\title{
Inflammatory myofibroblastic tumor of the uterus: a clinicopathological, immunohistochemical, and molecular analysis of 13 cases highlighting their broad morphologic spectrum
}

Jennifer A Bennett ${ }^{1}$, Valentina Nardi ${ }^{2}$, Marjan Rouzbahman ${ }^{3}$, Vicente Morales-Oyarvide ${ }^{4}$, G Petur Nielsen ${ }^{2}$ and Esther Oliva ${ }^{2}$

${ }^{1}$ Department of Pathology and Laboratory Medicine, Lahey Hospital and Medical Center, Burlington, MA, USA; ${ }^{2}$ Department of Pathology, Massachusetts General Hospital, Boston, MA, USA; ${ }^{3}$ Department of Laboratory Medicine and Pathobiology, Toronto General Hospital, Toronto, ON, Canada and ${ }^{4}$ Department of Medical Oncology, Dana Farber Cancer Institute, Boston, MA, USA

Inflammatory myofibroblastic tumors of the uterus are rare, and although most have a favorable prognosis, a small subset exhibit extrauterine disease, recur, or cause death. In this study, we evaluated the morphology and immunoprofile of 13 uterine inflammatory myofibroblastic tumors, including four with aggressive behavior. $A L K$ rearrangements were detected by fluorescence in situ hybridization and fusion partners by anchored multiplex assay. Patients ranged from 8 to 63 (mean 39) years and tumors from 2.5 to 20 (mean 7.4) cm. Myxoid, compact, and hyalinized patterns were noted in 13, 12, and 2 tumors, ranging from 1 to $100 \%, 5$ to $99 \%$, and 0 to $5 \%$, respectively. Nuclear atypia was mild in six (46\%), moderate in five (38\%), and severe in two (15\%), with ganglionlike cells in two tumors. Mitoses ranged from 0 to 24 (mean 5) per 10 high-power fields. Inflammation was mild in five $(38 \%)$, moderate in three $(23 \%)$, and marked in five $(38 \%)$, consisting of a lymphoplasmacytic infiltrate that was lymphocyte-predominant in six (46\%). Lymphovascular invasion was noted in two (15\%) and necrosis in eight (62\%). All but one tumor were ALK-positive by immunohistochemistry, with granular cytoplasmic staining in nine (82\%). ALK rearrangements (tested in 10) were detected in eight and was absent in one. The remaining tumor showed an isolated green $5^{\prime}$ ALK signal. Fusion partners were identified in $10(77 \%)$ and included $T H B S 1$ $(n=3)$, IGFBP5 $(n=2)$, DES $(n=2)$, SEC31 $(n=1), \operatorname{TPM3}(n=1)$, and TIMP3 $(n=1)$. Size $\geq 8 \mathrm{~cm}$ was predictive of aggressive behavior $(P<0.01)$, with increased mitoses ( $\geq 7$ per 10 high-power fields), lymphovascular invasion, and compact-predominance approaching statistical significance. These data show that inflammatory myofibroblastic tumors of the uterus are morphologically heterogenous with frequent ALK expression and a variety of $A L K$ fusion partners. Recognition of this rare mesenchymal neoplasm is crucial as those with aggressive behavior can potentially be treated with tyrosine kinase inhibitors.

Modern Pathology (2017) 30, 1489-1503; doi:10.1038/modpathol.2017.69; published online 30 June 2017

Inflammatory myofibroblastic tumor is a mesenchymal neoplasm, most commonly occurring in the lung or soft tissues of the abdomen, pelvis, and retroperitoneum. ${ }^{1}$ Most extrapulmonary inflammatory

Correspondence: Dr JA Bennett, MD, Department of Pathology, Lahey Hospital and Medical Center, 41 Mall Road, Burlington, MA 01805, USA.

E-mail: Jennifer.A.Bennett@Lahey.org

Received 27 April 2017; revised 8 May 2017; accepted 9 May 2017; published online 30 June 2017 myofibroblastic tumors are benign, but $25 \%$ recur locally, whereas $<2 \%$ metastasize. ${ }^{2}$ Approximately $50 \%$ of inflammatory myofibroblastic tumors harbor rearrangements involving the anaplastic lymphoma kinase $(A L K)$ gene on chromosome $2 \mathrm{p} 23$, but more recently, rearrangements with $R O S 1, P D G F R B, R E T$, and NTRK1 have been identified in a subset of $A L K$ negative tumors. ${ }^{3,4}$

Inflammatory myofibroblastic tumors are rare in the uterus with only 53 cases reported to date (Table 1). ${ }^{5-23}$ Extrauterine disease at the time of surgery was noted in 
Table 1 Previously reported cases of inflammatory myofibroblastic tumors of the uterus

\begin{tabular}{|c|c|c|c|c|c|c|c|c|c|}
\hline Reference & $\begin{array}{l}\text { \# of } \\
\text { Cases }\end{array}$ & $\begin{array}{c}\text { Age } \\
\text { (years) }\end{array}$ & Location & Extrauterine disease & Recurrence & Follow-up & Status & $A L K I H C$ & $A L K F I S H$ \\
\hline Gilks et al. ${ }^{5}$ & $2^{\mathrm{a}}$ & 6,30 & Corpus & No & No & $4.5,5.5$ years & NED & NP & NP \\
\hline $\begin{array}{l}\text { Abenoza } \\
\text { et al. }\end{array}$ & 1 & 58 & Cervix & No & No & 6 months & NED & $\mathrm{NP}$ & NP \\
\hline Kargi et al. ${ }^{7}$ & 1 & 53 & Corpus & No & No & 2 years & NED & $\mathrm{NP}$ & $\mathrm{NP}$ \\
\hline Azuno et al. ${ }^{8}$ & 1 & 58 & Corpus & No & N/A & N/A & N/A & Positive & NP \\
\hline Gucer et al. ${ }^{9}$ & 1 & 48 & Cervix & Parametria and vagina & No & 8 months & NED & NP & $\mathrm{NP}$ \\
\hline $\begin{array}{l}\text { Rabban } \\
\text { et al. } .^{10}\end{array}$ & $6^{\mathrm{a}}$ & $6-46$ & Corpus & Parametria $(n=1)$, No $(n=5)$ & No & $\begin{array}{c}1.5-5 \text { years }(n=4) \\
\text { N/A }(n=2)\end{array}$ & $\begin{array}{l}\text { NED }(n=4), \text { N/ } \\
\text { A }(n=2)\end{array}$ & $\begin{array}{l}\text { Positive }(n=5), \\
\text { NP }(n=1)\end{array}$ & NP \\
\hline $\begin{array}{l}\text { Shintaku } \\
\text { et al. }{ }^{11}\end{array}$ & 1 & 63 & Corpus & No & No & 8 months & NED & Positive & NP \\
\hline Gupta et al. ${ }^{12}$ & 1 & 14 & Corpus & No & No & 1 year & NED & $\mathrm{NP}$ & NP \\
\hline Olgan et al. ${ }^{13}$ & 1 & 28 & Corpus & No & N/A & $\mathrm{N} / \mathrm{A}$ & N/A & Positive & NP \\
\hline $\begin{array}{l}\text { Fuehrer } \\
\text { et al. }{ }^{14}\end{array}$ & 7 & N/A & $\begin{array}{l}\text { Corpus }(n=5) \text {, } \\
\text { cervix }(n=2)\end{array}$ & N/A & N/A & N/A & N/A & $\begin{array}{l}\text { Positive }(n=6), \\
\text { Negative }(n=1)\end{array}$ & $\begin{array}{l}\text { Positive }(n=5), \\
\text { Negative }(n=2)\end{array}$ \\
\hline Dong et al. ${ }^{15}$ & 1 & 19 & Corpus & No & Yes & $>4$ months & AWD & Positive & NP \\
\hline $\begin{array}{l}\text { Kushnir } \\
\text { et al. }{ }^{16}\end{array}$ & 1 & 30 & Corpus & $\begin{array}{l}\text { Fallopian tubes, ovaries, pelvic } \\
\text { sidewall, parametrium, bladder, } \\
\text { and rectosigmoid peritoneum }\end{array}$ & No & 6 months & NED & Negative & NP \\
\hline $\begin{array}{l}\text { Fraggetta } \\
\text { et al. }{ }^{17}\end{array}$ & 1 & 10 & Cervix & Pelvic lymph nodes & No & 20 months & NED & Positive & Positive \\
\hline $\begin{array}{l}\text { Parra-Herran } \\
\text { et al. }{ }^{18}\end{array}$ & 10 & $29-73$ & $\begin{array}{l}\text { Corpus }(n=8), \\
\text { cervix }(n=1) \text {, } \\
\text { corpus/cervix } \\
(n=1)\end{array}$ & Vagina $(n=1)$, No $(n=9)$ & $\begin{array}{l}\text { Yes }(n=2), \text { No } \\
(n=4), \text { N/A } \\
(n=4)\end{array}$ & $\begin{array}{l}2 \text { months- } 3 \text { years } \\
(n=6), \text { N/A }(n=4)\end{array}$ & $\begin{array}{l}\text { NED }(n=4) \\
\text { AWD }(n=2), \\
\text { N/A }(n=4)\end{array}$ & $\begin{array}{l}\text { Positive } \\
(n=10)\end{array}$ & $\begin{array}{l}\text { Positive }(n=8), \mathrm{NP} \\
(n=2)\end{array}$ \\
\hline $\begin{array}{l}\text { Subbiah } \\
\text { et al. }{ }^{19}\end{array}$ & 1 & $50 \mathrm{~s}$ & N/A & No & Yes & $>3$ years & AWD & Positive & NP \\
\hline $\begin{array}{l}\text { Parra-Herran } \\
\text { et al. }\end{array}$ & 4 & $53-56$ & N/A & No & N/A & $\begin{array}{c}2-4 \text { years }(n=2) \\
\text { N/A }(n=2)\end{array}$ & $\begin{array}{l}\text { DOD }(n=2), \mathrm{N} / \\
\mathrm{A}(n=2)\end{array}$ & Positive $(n=4)$ & $\begin{array}{l}\text { Positive }(n=2), \\
\text { Negative }(n=1), \mathrm{NP} \\
(n=1)\end{array}$ \\
\hline Sholl et al. ${ }^{21}$ & 1 & 53 & Corpus & No & Yes & Not specified & NED & NP & Positive \\
\hline Wojcik $^{22}$ & 1 & 30 & Corpus & No & N/A & N/A & N/A & Positive & Positive \\
\hline $\begin{array}{l}\text { Haimes } \\
\text { et } a l^{23}\end{array}$ & 12 & $24-78$ & N/A & No & $\begin{array}{l}\text { No }(n=11), \text { N/A } \\
(n=1)\end{array}$ & $\begin{array}{l}\text { 9-93 months } \\
(n=11), \mathrm{N} / \mathrm{A} \\
(n=1)\end{array}$ & $\begin{array}{l}\text { NED }(n=11), \\
\text { N/A }(n=1)\end{array}$ & $\begin{array}{l}\text { Positive } \\
(n=11) \\
\text { Negative }(n=1)\end{array}$ & $\begin{array}{l}\text { Positive }(n=4), \\
\text { Negative }(n=3), \\
\text { Failed }(n=1), \text { NP } \\
(n=4)\end{array}$ \\
\hline
\end{tabular}

Abbreviations: AWD, alive with disease; DOD, dead of disease; FISH, fluorescence in situ hybridization; N/A, not available; NED, no evidence of disease; NIHC, immunohistochemistry; NP, not performed.

Same case used in both studies (6-year-old patient). 
five, ${ }^{9,10,16-18}$ and when follow-up was available $(n=34),{ }^{5-7,9-12,15-21,23}$ recurrences occurred in five patients, $15,18,19,21$ whereas two others died of disease. $^{20}$ In one study, those that recurred were myxoid-predominant with tumor cell necrosis and elevated mitotic activity compared to those with a benign clinical course. ${ }^{18}$ When ALK immunohistochemistry and fluorescence in situ hybridization was performed, 93\% (43/46) were ALK-positive and 79\% (22/28) ALK-rearranged. ${ }^{8,10,11,13-23}$

Although smooth muscle and endometrial stromal tumors are the most common uterine mesenchymal neoplasms, their morphology may overlap with that seen in inflammatory myofibroblastic tumors. The frequent expression of desmin and CD10 in the latter further contributes to the diagnostic challenge. Herein, we evaluate the morphology, immunoprofile, and molecular phenotype of a series of uterine inflammatory myofibroblastic tumors, as well as features potentially predictive of behavior.

\section{Materials and methods}

Thirteen inflammatory myofibroblastic tumors of the uterus were identified from the archives at the Massachusetts General Hospital and Lahey Hospital and Medical Center, as well as the consultation files of one of the authors (EO). Age, clinical presentation, type of surgery (hysterectomy, myomectomy), recurrences, and date/status at last follow-up were obtained from the medical records or submitting pathologist. Macroscopic features were recorded from the pathology reports.

\section{Pathological Examination}

The number of hematoxylin and eosin-stained tumor slides ranged from 1 to 27 (mean 8). Tumor border was classified as well-circumscribed, focally irregular (rare small tongues or islands of tumor present on only one slide and located $<2 \mathrm{~mm}$ from the otherwise well-circumscribed mass), or infiltrative. Each tumor was examined to determine the percentages of the three previously described histological patterns-myxoid, compact, and hyalinized. ${ }^{24}$ Briefly, the myxoid pattern was comprised of loosely arranged spindle cells set in a myxoid/edematous background associated with a prominent vasculature. The compact pattern had densely arranged spindle cells with fascicular or storiform architecture, whereas the hyalinized pattern consisted of sparsely cellular collagen, resembling a scar or fibromatosis.

Nuclear atypia was graded as mild, moderate, or severe. Mild atypia was defined as uniform cells with a nuclear to cytoplasmic ratio $<1$ to 3 . Nuclei were elongated with smooth contours, inconspicuous nucleoli, and vesicular chromatin. For moderate atypia, the nuclear to cytoplasmic ratio was between 1 to 3 and 1 to 2 . Nuclei were ovoid, plump, and hyperchromatic with mildly irregular contours and occasional nucleoli. In severe atypia, cells were markedly pleomorphic with a nuclear to cytoplasmic ratio $>1$ to 2 and prominent nucleoli. Only the highest grade was recorded, and had to occupy at least one high-power field $(\times 400)$. The presence of ganglion-like cells (ovoid cells with eccentric, indented, and convoluted nuclei, prominent nucleoli, and eosinophilic cytoplasm), vessel morphology, mitotic index per 10 high-power fields, necrosis, and lymphovascular invasion were also noted.

The predominant location of inflammation (myxoid, compact, hyalinized, or equally distributed) as well as type of inflammatory cells was recorded along with extent (diffuse-present on at least 50\% of slides or focal-present on $<50 \%$ of slides). The density of inflammation was graded as mild (evident at highpower, 200X), moderate (evident at medium-power, $\times 100$ ), or marked (evident at low-power, $\times 40$ ).

\section{Immunohistochemistry}

In tumors where immunohistochemistry had not been performed, primary monoclonal antibodies to ALK-1 (clone 5A4 ER2, ready to use; Leica Biosystems, Buffalo Grove, IL, USA), desmin (clone DE-R-11 ER2, ready to use; Leica Biosystems), CD10 (clone 56C6 ER2, ready to use; Leica Biosystems), and h-caldesmon (clone h-CD ER1, dilution 1:100; Dako, Santa Clara, CA, USA) were applied to a representative $5 \mu \mathrm{m}$-thick section of formalin-fixed, paraffinembedded tumor. Stains were interpreted as negative (0), rare positivity (1+), multifocal $(2+)$, or diffuse $(3+)$. The pattern of ALK staining was also noted.

\section{Fluorescence In Situ Hybridization}

$A L K$ rearrangement was identified by fluorescence in situ hybridization testing. Briefly, $5 \mu$ m-thick sections of formalin-fixed, paraffin-embedded tumor material were prepared, and an H\&E section reviewed to select regions for hybridization that contained a majority of tumor cells. An $A L K$ breakapart probe (Kreatech ALK Breakapart FISH Proximal Green and Distal Red probes) was hybridized, and used to calculate the number of cells out of 50 scored containing a rearrangement. An $A L K$ rearrangement was reported if more than $15 \%$ of cells showed split signals and was confirmed with the $A L K$ break-apart probe (Vysis LSI ALK (2p23) Dual Color, Break Apart Rearrangement Probe). Almost invariably, $A L K$ translocations resulted in chimeric proteins containing a $5^{\prime}$-end partner fused to the $A L K$ tyrosine kinase domain at the $3^{\prime}$-end.

\section{Anchored Multiplex Polymerase Chain Reaction}

For targeted fusion-transcript detection of tumor RNA, anchored multiplex polymerase chain reaction using the ArcherDx FusionPlex Solid Tumor Panel 
and next-generation sequencing using Illumina Nextseq (San Diego, CA, USA) was used as previously described. ${ }^{25}$ Briefly, nucleic acid was reverse-transcribed with random hexamers, followed by second-strand synthesis to create double-stranded complementary DNA to be end-repaired, adenylated, and ligated with a half-functional adapter. Two hemi-nested polymerase chain reactions using the ArcherDx FusionPlex Solid Tumor Kit primers were performed to create a sequencing library targeting specific exons (for $A L K$ exons 1, 3, 17, 19-22, and 29). A laboratory-developed algorithm was used for fusion-transcript detection and annotation.

\section{Statistical Analysis}

The associations between clinical course (benign vs aggressive behavior) and clinicopathological characteristics were assessed in univariate analyses using the Fisher's exact test and $\chi^{2}$ test for categorical variables and the Wilcoxon rank-sum test for continuous variables. We conducted two secondary univariate analyses of clinicopathological features comparing (1) histologically banal tumors that did not recur against histologically malignant tumors, and (2) histologically banal tumors that did not recur against histologically banal tumors that did recur. We built receiver-operator curves to assess the performance of selected continuous variables to identify features of interest (eg, aggressive clinical behavior). All hypothesis tests were two-sided and statistical significance was set at $P<0.05$. Statistical analyses were performed using Stata (Version 14.0, StataCorp, College Station, TX, USA).

\section{Results}

\section{Clinical Features}

Patients' age ranged from 8 to 63 (mean 39, median 36) years. Clinical presentation was non-specific and included presumed fibroids $(n=4)$, abnormal uterine bleeding $(n=3)$, pelvic mass $(n=2)$, low back pain $(n=1)$, and abdominal pain $(n=1)$. It was an incidental finding at cesarean section in two patients. Total hysterectomy was performed in seven patients, myomectomy in five, and supracervical hysterectomy in one. Clinical features are highlighted in Table 2.

\section{Pathological Examination}

All inflammatory myofibroblastic tumors arose in the corpus. When known $(n=10)$, the mass was submucosal $(6 / 10,60 \%)$, transmural with cervical involvement $(1 / 10,10 \%)$, intramural $(1 / 10,10 \%)$, subserosal with adhesions to the bowel $(1 / 10,10 \%)$, or involved both the endometrium and myometrium $(1 / 10,10 \%)$. Tumors ranged from 2.5 to 20 (mean 7.4, median 5.5) $\mathrm{cm}$. Size was unavailable in one 


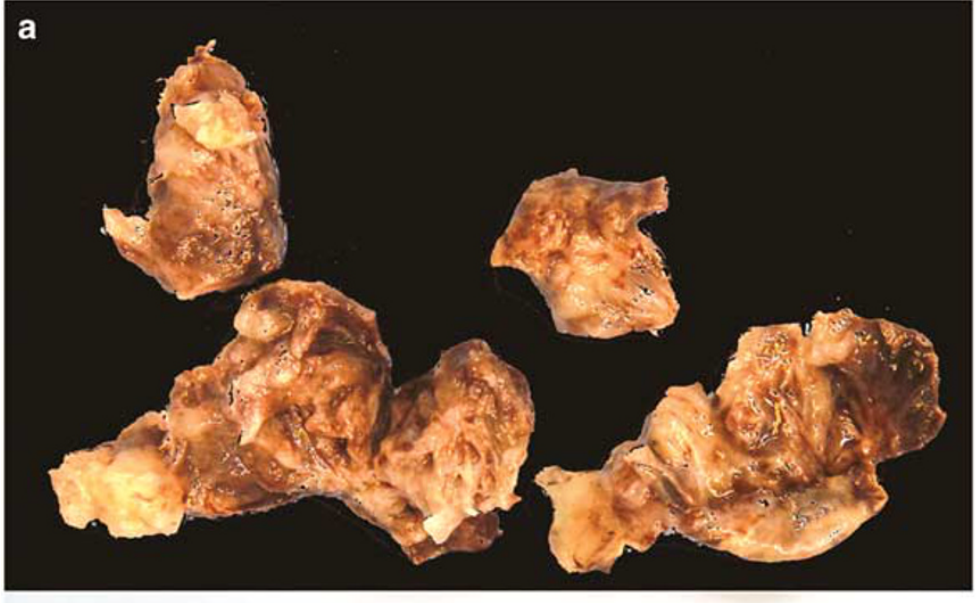

$\begin{array}{lllllllllllll}160 & 170 & 180 & 190 & 200 & 210 & 220 & 230 & 240 & 250 & 260 & 270 & 286\end{array}$

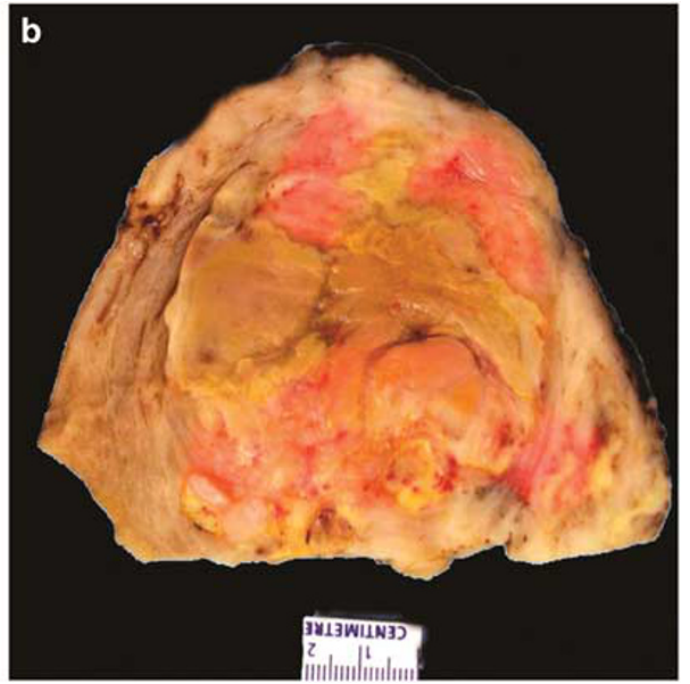

Figure 1 Uterine inflammatory myofibroblastic tumor. The cut surface ranged from tan-brown and gelatinous (a) to tan-white, lobulated, and extensively necrotic (b).

case. Most $(8 / 13,62 \%)$ were tan, pink, or white, whereas three had interspersed yellow foci, and in two, the color was not described. Other features noted included a soft consistency $(5 / 13,38 \%)$, whorling appearance $(5 / 13,38 \%)$, hemorrhage $(4 / 13$, $31 \%)$, necrosis $(4 / 13,31 \%)$ (Figure $1 \mathrm{~b}$ ), and cyst formation $(2 / 13,15 \%)$. Five tumors $(38 \%)$ were described as mucinous, myxoid (Figure 1a), or edematous.

Tumors from hysterectomy specimens showed an infiltrative border in $75 \%(6 / 8)$ and a focally irregular border in $25 \%$ (2/8). Infiltrative tumors showed three main patterns-nodular/tongue-like invasion similar to endometrial stromal sarcomas (3/6, $50 \%)$, a jagged sawtooth appearance $(2 / 6,33 \%)$, or percolation of tumor cells into the myometrium $(1 / 6,17 \%)$. Adjacent myometrium was not present in any of the myomectomy specimens; however, two patients subsequently had a hysterectomy that showed either no residual tumor (case 6) or a necrotic lesion without any invasion into the myometrium (case 11).

A myxoid pattern was observed in all 13 tumors, which comprised from one to $100 \%$ of the mass (mean 54\%, median 70\%). This pattern was universally hypocellular with some tumors showing areas resembling nodular fasciitis (7/13, 54\%), whereas others consisted of acellular myxoid pools $(4 / 13,31 \%)$ or pseudocysts containing myxoid material $(3 / 13,23 \%)$ (Figure 2a-c). Occasionally, cells in the myxoid foci were arranged in a storiform (6/13, 46\%) (Figure 2d) and/or fascicular (5/13, 38\%) pattern. All but one tumor showed a compact pattern, comprising $5-99 \%$ of the mass (mean $46 \%$, median $30 \%$ ). These hypercellular regions were primarily fascicular, but $17 \%(2 / 12)$ showed a storiform appearance (Figure 2e and f). The cells had a smooth muscle appearance in $42 \%$ (5/12), whereas in one other tumor (case 5) cells resembled endometrial stroma (Figure $2 \mathrm{~g}$ and $\mathrm{h}$ ). The hyalinized pattern was only noted in $15 \%(2 / 13)$ and comprised a minimal amount $(5 \%)$ of these tumors. These foci, which were hypocellular with dense collagen bands (Figure 2i) and a rare collagenous micronodule (case 4), were adjacent to a compact pattern.

Nuclear atypia was mild in $46 \%(6 / 13)$, moderate in $38 \%(5 / 13)$, and severe in $15 \%(2 / 13)$, with ganglionlike cells noted in 15\% (2/13) (Figure 3a-d). Mitotic activity ranged from 0 to 24 (mean 5 , median 2) per 10 high-power fields. An unexpected morphological feature was noted in two tumors, both of which were incidentally identified at cesarean section. In these lesions, the spindle cells had a decidual-like appearance, one of which was quite striking (Figure 3f).

The inflammatory cells were primarily located in the compact foci in $46 \%(6 / 13)$, equally distributed between compact and myxoid patterns in $31 \%$ (4/13), and myxoid-predominant in $23 \%(3 / 13)$. Inflammation was diffuse in all tumors with mild inflammation in $38 \%(5 / 13)$, moderate in $23 \%$ (3/13), and marked in 38\% (5/13) (Figure 4a-d). Although a lymphoplasmacytic infiltrate was noted in all tumors, $46 \%$ (6/13) were lymphocytepredominant. Other inflammatory cells observed included foamy histiocytes $(5 / 13,38 \%)$, neutrophils (4/13, 31\%), eosinophils $(3 / 13,23 \%)$, and Touton giant cells $(2 / 15,15 \%)$ (Figure 3e). Small lymphoid aggregates, which formed germinal centers were noted in one tumor, whereas plasma cell clusters were identified in another. Thin-walled and elongated vessels were prominent in all tumors, whereas scattered thick-walled vessels, reminiscent of a leiomyoma, were noted in $46 \%(5 / 13)$, staghorn vessels in $23 \%(3 / 13)$, and a chicken wire-like vasculature in $8 \%(1 / 13)$ (Figure $4 \mathrm{e}-\mathrm{h})$. Small 

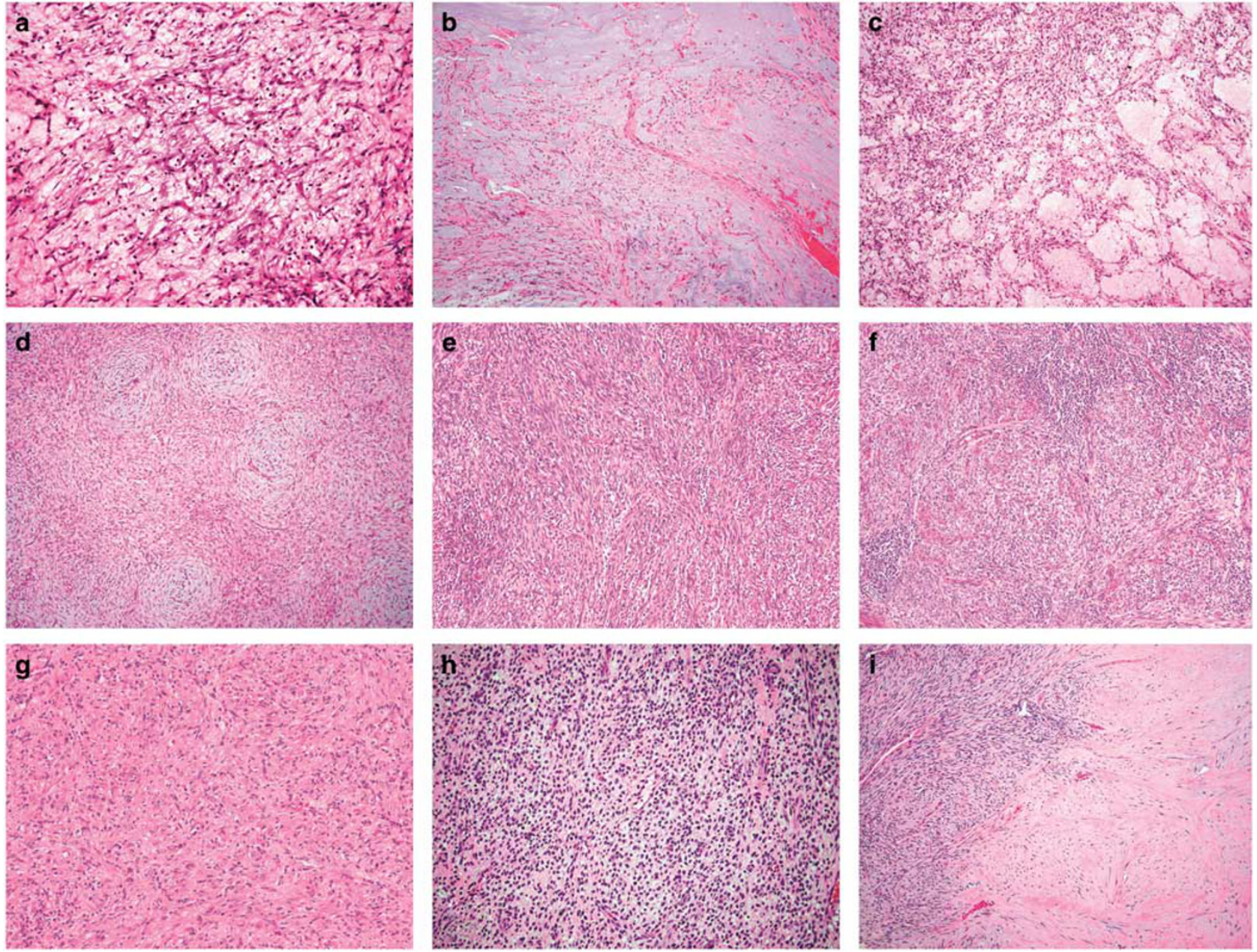

Figure 2 Uterine inflammatory myofibroblastic tumor. The myxoid pattern had variable appearances including: myxoid stroma being distributed among spindled cells and inflammatory cells resulting in a nodular fasciitis-like morphology (a), large acellular pools (b), pseudocysts (c), or occasionally as a background within a storiform growth of tumor cells (d). The compact pattern showed either a fascicular (e) or storiform (f) architecture. In some tumors, the appearance closely mimicked that of a smooth muscle tumor (g), and much less frequently, cells showed diffuse growth resembling an endometrial stromal neoplasm (h). The hyalinized pattern showed abundant collagen deposition, resembling a scar (i).

arterioles were not observed in any tumors. Necrosis was present in $62 \%(8 / 13)$ and lymphovascular invasion in $15 \%(2 / 13)$. Morphologic features are highlighted in Table 3.

Extrauterine disease at time of initial diagnosis was noted in two patients. In case 4, tumor involved the ovaries, fallopian tubes, and omentum, and was comprised exclusively of sheets of markedly atypical epithelioid cells with a diffuse lymphocytepredominant infiltrate. In case 8 , tumor involved the right adnexal soft tissue, cul-de-sac, and pelvic sidewall. Both myxoid and compact patterns were observed, which consisted of spindled and epithelioid cells with variable nuclear atypia in a lymphocyte-predominant infiltrate.

\section{Immunohistochemistry}

Most (10/13, 77\%) inflammatory myofibroblastic tumors had $3+$ expression of ALK; however, the remaining three showed $0,1+$, or $2+$. Four ALK staining patterns were observed-granular cytoplasmic staining $(8 / 11,73 \%)$, granular cytoplasmic with distinct staining of the cytoplasmic membrane $(1 / 11$, $9 \%)$, smooth cytoplasmic $(1 / 11,9 \%)$, and smooth cytoplasmic with perinuclear accentuation (1/11, $9 \%$ ) (Figure 5a-d). The staining pattern for one tumor was not known. CD10 and desmin were performed in 12 and 11 tumors, respectively, with most showing 2+ (4 each for CD10 and desmin) or 3+ (6 for CD10 and 5 for desmin) expression. Caldesmon, performed in 12 tumors, was only notable in three, and was primarily restricted to the compact areas. Two of these were compact-predominant (70 and $75 \%$, respectively), whereas the third was myxoid-predominant $(30 \%)$, but had compact foci with a striking smooth muscle appearance. Slides representative of the hyalinized pattern were not stained for the above markers due to the focality of this pattern. Immunohistochemical results are illustrated in Table 4. 


\section{Fluorescence In Situ Hybridization}

Fluorescence in situ hybridization for $A L K$ rearrangements was successfully performed in 10 inflammatory myofibroblastic tumors (Table 4). Rearrangements were detected in eight tumors (Figure 5e) and was absent in one. In the remaining tumor, an abnormal pattern characterized by a single-isolated green $5^{\prime}$ $A L K$ probe (in addition to a single normal unsplit redgreen pair), was noted (Figure $5 \mathrm{f}$ ).
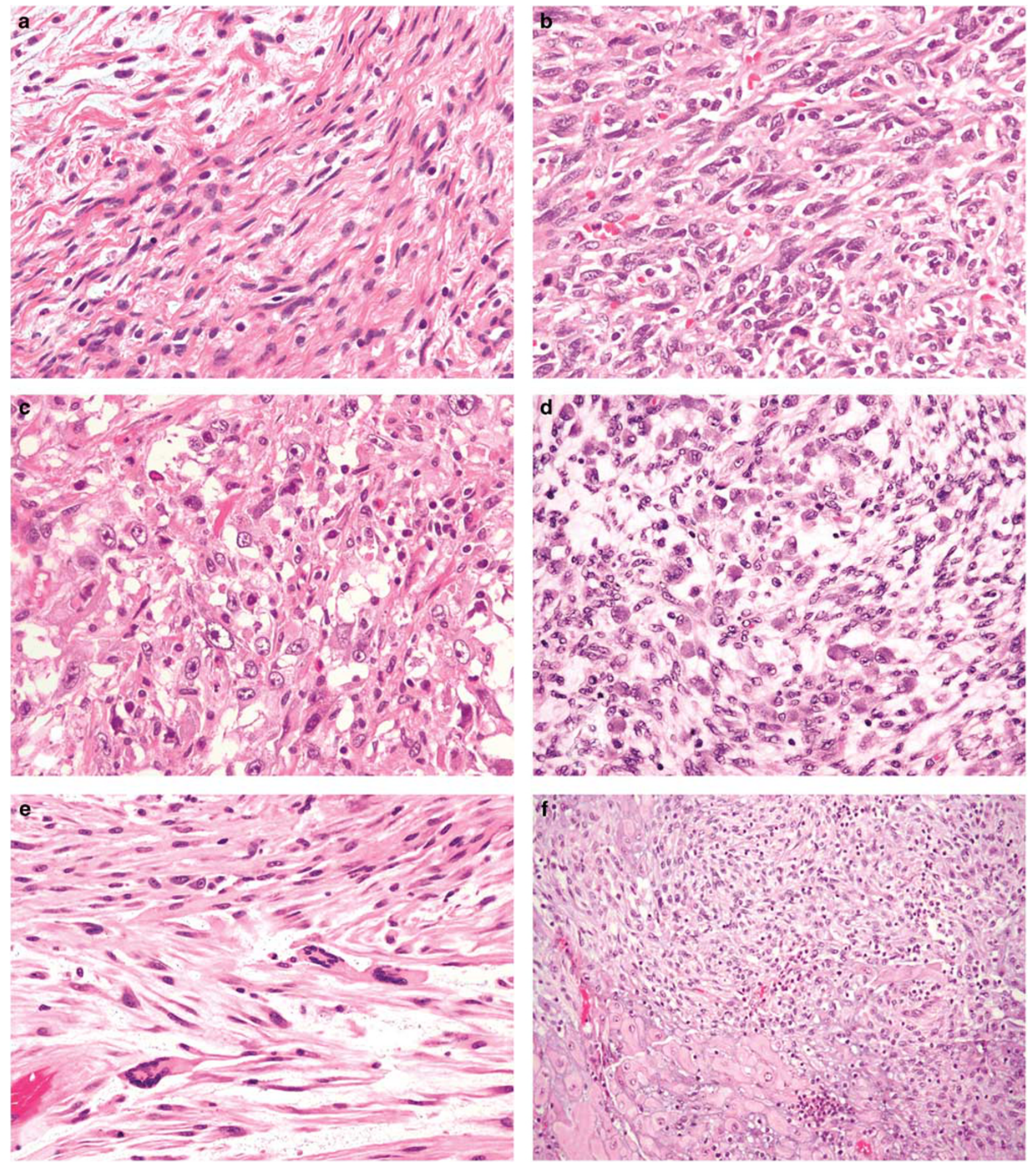

Figure 3 Uterine inflammatory myofibroblastic tumor. In tumors with mild atypia, cells were uniform with a low nuclear to cytoplasmic ratio, elongated nuclei, and inconspicuous nucleoli (a). Cells with moderate atypia were plump with hyperchromatic nuclei and occasional nucleoli (b), whereas in severe atypia, cells were markedly pleomorphic with a high nuclear to cytoplasmic ratio and prominent nucleoli (c). Ganglion-like cells (d), touton cells containing multinucleation in a wreath-like fashion (e), and myofibroblastic cells merging with decidua (f) were rarely noted. 

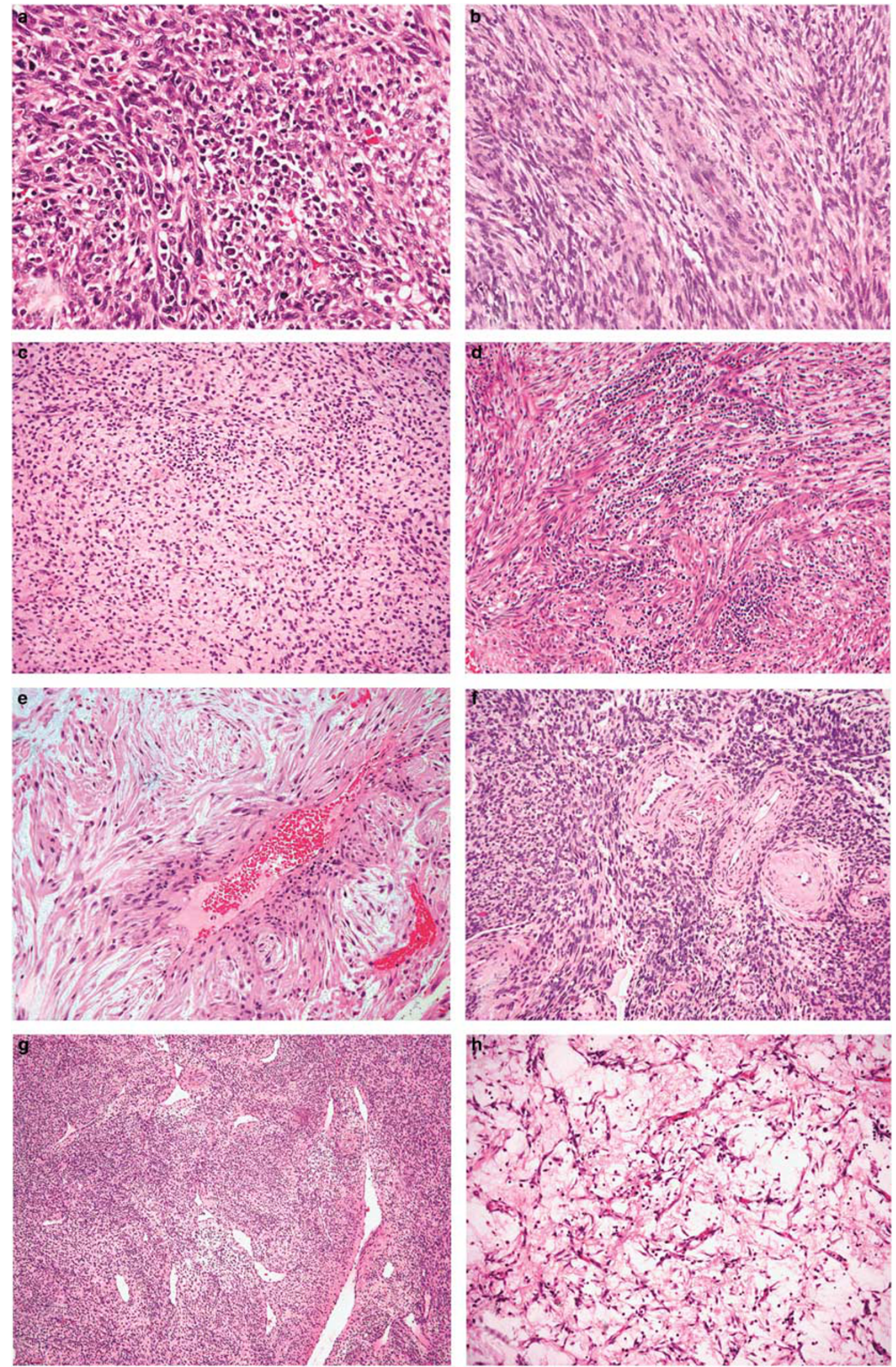

Figure 4 Uterine inflammatory myofibroblastic tumor. All tumors had a lymphoplasmacytic infiltrate (a). They either showed sparse inflammation noticeable only at high-power $(\times 200)(\mathbf{b})$, moderate (more conspicuous) density of inflammation present at medium-power $(\times 100)(\mathbf{c})$, or marked inflammation that was obvious on low-power examination $(\times 40)(\mathbf{d})$. Thin-walled and elongated vessels were a constant feature (e), whereas thick-walled vessels (f), staghorn vessels (g), or chicken wire-like vasculature (h) were noted in some tumors. 


\section{Anchored Multiplex Assay}

The anchored multiplex assay was performed in all 13 inflammatory myofibroblastic tumors and an ALK fusion was detected in $77 \%$ (Table 4). Fusion partners included THBS1 $(n=3)$, IGFBP5 $(n=2)$, DES $(n=2)$, SEC31 $(n=1)$, TPM3 $(n=1)$, and TIMP3 $(n=1)$. Of note, one of the DES-ALK fusions (case 4) was of low quality and only three fusion reads were identified. In the remaining $23 \%$, an $A L K$ fusion was not detected, but in one, quality was suboptimal (case 2).

\section{Follow-Up}

Follow-up was available for 12 patients and ranged from 1 to 132 (mean 31, median 7) months with eight patients being alive and well, one alive with disease, and three dead of disease. Multiple recurrences occurred in two patients $(17 \%)$. In one patient (case 2 ), the first occurred at the vaginal apex at 3.4 years, and the second at the vaginal apex and ileum at 8.8 years after initial diagnosis. She ultimately succumbed to her disease after 11 years. Another patient (case 9) had three recurrences; localized to the abdomen 1 month after surgery, ovary at 10 months, and an unspecified site at 1 year. No additional follow-up is available. In the two patients with extrauterine disease at time of diagnosis, one (case 4) developed shortness of breath, a wound infection, and intestinal obstruction postoperatively. CT scan showed pulmonary metastases and she died 1 month following surgery. The other patient (case 8) was readmitted for nausea, vomiting, and progressive pelvic sidewall disease 2 months after surgery and died 2 months thereafter.

\section{Statistical Analysis}

On univariate analysis, only size $\geq 8 \mathrm{~cm}$ (sensitivity and specificity $100 \%$ ) was found to be significantly associated with aggressive behavior. A mitotic index $\geq 7$ per 10 high-power fields (sensitivity $75 \%$, specificity $100 \%$ ), presence of lymphovascular invasion, and compact-predominance (sensitivity $75 \%$, specificity 67\%) approached statistical significance.

A secondary analysis comparing histologically banal tumors that did not recur with histologically malignant ones showed size $\geq 10.5 \mathrm{~cm}$ (sensitivity and specificity $100 \%$ ), severe atypia, mitoses $\geq 18 / 10$ high-power fields (sensitivity and specificity 100\%), and lymphovascular invasion to be predictive of aggressive behavior. Multivariate analysis was not able to be performed due to the small sample size. In another secondary analysis, comparing histologically banal tumors that did not recur to histologically banal ones that recurred only size $\geq 8 \mathrm{~cm}$ (sensitivity and specificity $100 \%$ ) was significant of aggressive behavior. Univariate analyses with associated $P$-values are shown in Supplementary Table 1. 

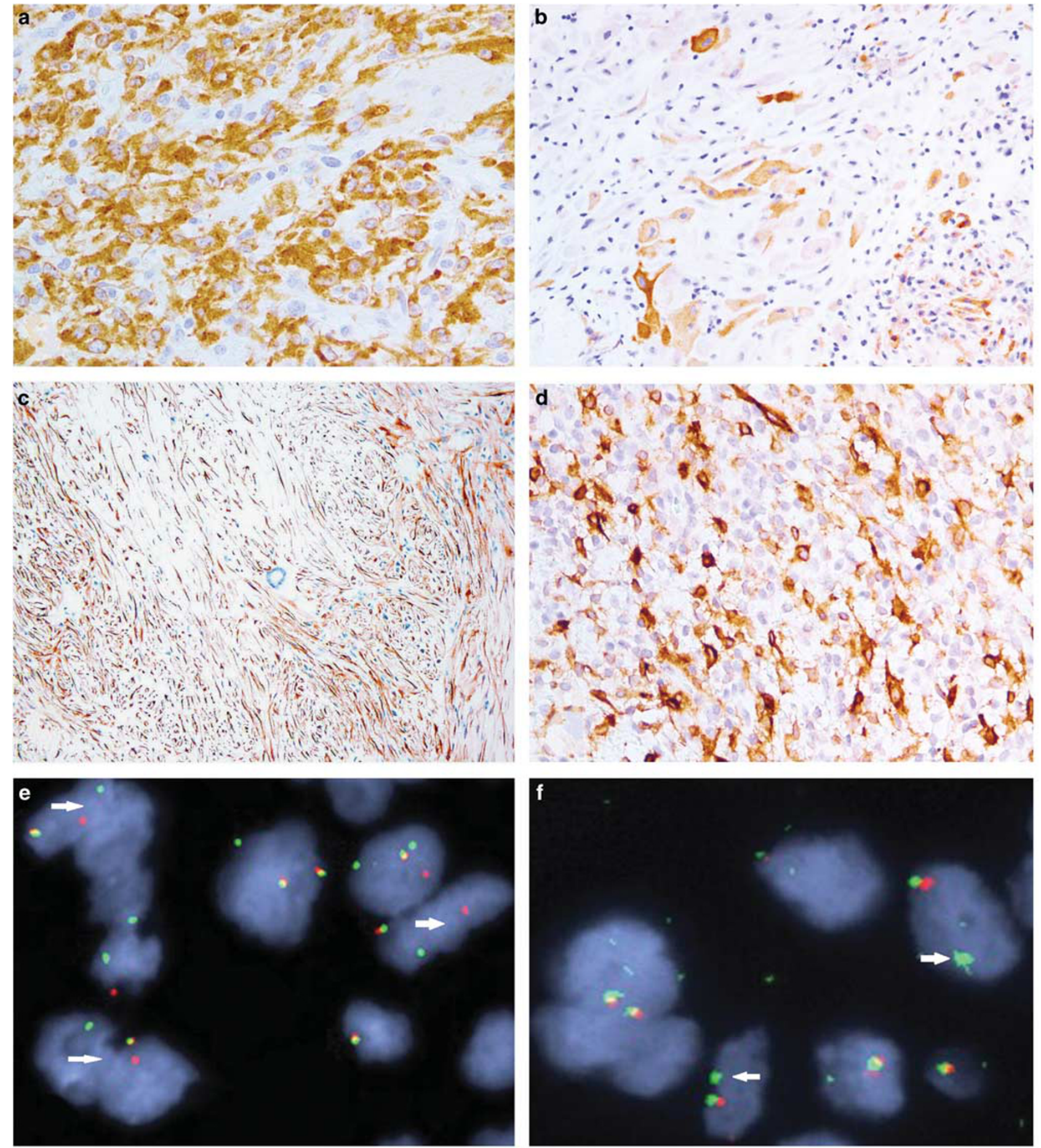

Figure 5 Uterine inflammatory myofibroblastic tumor. Granular cytoplasmic ALK staining was noted in most tumors (a) with one also showing distinct staining of the cytoplasmic membrane (b). Smooth cytoplasmic staining (c) was seen in two other tumors, one of which also had perinuclear accentuation (d). The classic $A L K$ rearrangement was seen in $80 \%$ (e). However, one tumor showed an isolated green $\left(5^{\prime}\right)$ probe, in addition to a single normal unsplit red-green pair (f).

\section{Clinicopathologic Features of Aggressive Tumors}

Aggressive inflammatory myofibroblastic tumors were more common in older patients (mean 42 vs 37 years, $P=0.44$ ), larger (mean 13 vs $4.4 \mathrm{~cm}$, $P<0.01$ ), and compact-predominant (69 vs $35 \%$, $\mathrm{p}=0.09$ ). Severe nuclear atypia and lymphovascular invasion were only noted in aggressive tumors (both $P=0.08$ ), and mean mitotic count was higher (12.5 vs 2 per 10 high-power fields, $P=0.07$ ). Necrosis was present in all aggressive tumors, but in only $44 \%$ of benign tumors $(P=0.11)$. 
Table 4 Immunohistochemical and molecular findings of uterine inflammatory myofibroblastic tumors

\begin{tabular}{|c|c|c|c|c|c|c|}
\hline Case & $A L K$ & $C D 10$ & Desmin & Caldesmon & FISH & Anchored multiplex assay \\
\hline 1 & $3+$ & $2+$ & $3+$ & $3+$ & Rearranged & IGFBP5-ALK \\
\hline 2 & $1+$ & $3+$ & $2+$ & $2+$ & Rearranged & Negative (suboptimal) \\
\hline 3 & $3+$ & $2+$ & $2+$ & 0 & Failed & TIMP3-ALK \\
\hline 4 & 0 & $3+$ & 0 & 0 & Rearranged & DES-ALK (3 reads only) \\
\hline 5 & $3+$ & $2+$ & $3+$ & 0 & Rearranged & Negative \\
\hline 6 & $3+$ & $3+$ & $2+$ & $1+$ & Abnormal 'Green only' signal & Negative \\
\hline 7 & $3+$ & $2+$ & $3+$ & 0 & Rearranged & IGFBP5-ALK \\
\hline 8 & $3+$ & $3+$ & $1+$ & $1+$ & Not rearranged & SEC31-ALK \\
\hline 9 & $3+$ & 0 & $2+$ & 0 & NP & TPM3-ALK \\
\hline 10 & $3+$ & NP & NP & NP & Rearranged & THBS1-ALK \\
\hline 11 & $3+$ & $3+$ & NP & $3+$ & Rearranged & THBS1-ALK \\
\hline 12 & $3+$ & $1+$ & $3+$ & 0 & NP & DES-ALK \\
\hline 13 & $2+$ & $3+$ & $3+$ & 0 & Rearranged & THBS1-ALK \\
\hline
\end{tabular}

Abbreviations: FISH, fluorescence in situ hybridization; NP, not performed; 3+, diffuse; 2+, multifocal; 1+, rare; 0, negative.

\section{Discussion}

Herein we describe the morphology, immunoprofile, and molecular phenotype of the largest series of uterine inflammatory myofibroblastic tumors to date including four with aggressive behavior. In the literature, $21 \%$ of uterine inflammatory myofibroblastic tumors have shown an adverse outcome, characterized by extrauterine disease, recurrence, or death, $, 10,15-21$ which is slightly lower than the $31 \%$ identified in this study. Parra-Herran et al. ${ }^{18}$ showed that such tumors occurred in older patients (56 vs 38 years), were larger (12.3 vs $3.5 \mathrm{~cm})$, and myxoidpredominant with tumor cell necrosis and increased mitoses (mean 12.6 vs 3/10 high-power fields). Our results were similar with respect to patient age, size, and mitotic index, but most $(75 \%)$ of our aggressive tumors were compact-predominant. All of these tumors also had necrosis, but in contrast to the prior study, this feature was not limited to aggressive tumors. Severe nuclear atypia and lymphovascular invasion were also noted in a subset of our tumors with an adverse outcome, two features that were not identified in any tumors from the Parra-Herran et al. study. ${ }^{18}$

Extrauterine disease at time of diagnosis was present in two patients in this study. Disease was limited to the adnexal soft tissue, cul-de-sac, and pelvic sidewall in one (case 8), whereas in the other (case 4), extensive extrauterine disease was present including liver and lungs. Both patients died from disease, 1 and 4 months after surgery. Five inflammatory myofibroblastic tumors have also been reported in the literature to have extrauterine disease; ${ }^{9,10,16-18}$ however, two were lost to followup. ${ }^{10,18}$ Extrauterine disease in those with follow-up consisted of involvement of fallopian tubes, ovaries, pelvic sidewall, bladder, and rectosigmoid peritoneum, and parametria in one, ${ }^{16}$ involvement of parametria and vagina in another, ${ }^{9}$ and metastasis to pelvic lymph nodes in the third. ${ }^{17}$ The second patient had an inflammatory myofibroblastic tumor of cervical origin and reported a supracervical hysterectomy for benign disease several years earlier. In all three patients, no gross residual disease was noted at the end of surgery, and all were alive without disease 6,8 , and 20 months later. Due to the limited number of uterine inflammatory myofibroblastic tumors with extrauterine disease combined with little to no follow-up available, it is difficult to draw conclusions about prognosis of such patients. In this study, they both demonstrated a poor prognosis with death shortly after surgery. The three patients reported in the literature with a favorable prognosis had relatively brief follow-up, likely too short to reach such conclusion given that one of our patients without extrauterine disease (case 2), experienced several recurrences, the first occurring more than three years after original diagnosis.

Recurrences also occurred in two patients in this study. In one patient (case 2), tumor first recurred locally at the vaginal apex (3.4 years after surgery), and then recurred again, both locally (vaginal apex) and distantly (ileum), 5.4 years after the first recurrence. The other patient (case 9) first had a local recurrence 1 month after surgery; however, this could be due to subtotal resection as the operative details were unavailable. The second recurrence was in the ovary 9 months later, followed by a third recurrence at an unknown site during the following year. Only five inflammatory myofibroblastic tumors in the literature have recurred, ${ }^{15,18,19,21}$ most with a relatively short or unknown follow-up period. Of note, one patient who experienced multiple recurrences showed partial response to crizotinib chemotherapy and is currently alive with disease $>3$ years after initial diagnosis. ${ }^{19}$

Two inflammatory myofibroblastic tumors were incidentally discovered at the time of cesarean section and removed by myomectomy. In both of these the spindle cells had a decidual-like appearance, one of which was quite striking. Examination of the placenta showed no involvement by tumor in one patient, whereas details were not available for 
the other. Decidual-like change in inflammatory myofibroblastic tumors was previously reported by Banet et al. ${ }^{26}$ who described two cases arising in the placenta. Both tumors were confined to the placental disc and had morphologic features consistent with an inflammatory myofibroblastic tumor, although one was ALK-negative. More recently, Wojcik ${ }^{22}$ described a $10 \mathrm{~cm}$ mass between the rectum and posterior uterus that was incidentally identified at the time of cesarean section and removed by blunt dissection. The spindle cells showed a florid decidual-like appearance, that, in combination with the other morphologic features, ALK expression by immunohistochemistry, and $A L K$ rearrangement by fluorescence in situ hybridization, were diagnostic of an inflammatory myofibroblastic tumor. Haimes et $a .^{23}$ also described two inflammatory myofibroblastic tumors identified in pregnancy, one of which showed focal deciduoid changes. This tumor was ALK-positive by immunohistochemistry and ALKnegative by fluorescence in situ hybridization with an FN1-ALK fusion.

ALK immunohistochemistry using the Leica 5A4 clone was performed in all 13 inflammatory myofibroblastic tumors, 12 of which were positive. Interestingly, the one tumor that was negative (case 4), did have an $A L K$ rearrangement and a $D E S-A L K$ fusion. The latter was only noted in three fusion reads rather than the desired minimum of 10 , but this was attributed to suboptimal tissue quality. Potentially usage of the Ventana D5F3 clone, reported to be more sensitive and specific than the Leica 5A4 clone, ${ }^{27,28}$ could have resulted in ALK expression in our negative tumor. Nevertheless, the morphologic features and molecular results in this particular case supported the diagnosis of an inflammatory myofibroblastic tumor. However, the absence of ALK positivity in a lesion morphologically suggestive of an inflammatory myofibroblastic tumor should prompt the usage of other testing modalities including a different ALK antibody, fluorescence in situ hybridization, next-generation sequencing, or immunostaining for non- $A L K$ rearrangements (ROS1). Although lack of ALK staining is rare in uterine inflammatory myofibroblastic tumors, it has been reported in three cases. ${ }^{14,16,23}$ Interestingly, in two of these, the DAKO ALK-1 clone was used, ${ }^{14,23}$ whereas the clone was not specified in the third. ${ }^{16}$ Of note, two studies reported use of the Ventana D5F3 clone and all tested tumors $(n=14)$ were ALK-positive. ${ }^{18,20}$

Other studies have reported that the pattern and localization of ALK expression in extrauterine inflammatory myofibroblastic tumors may possibly correlate with specific gene fusions. ${ }^{1}$ Granular cytoplasmic staining was seen in all our tumors with an identifiable fusion partner, which is similar to other uterine inflammatory myofibroblastic tumors in the literature. ${ }^{10,11,13,14,18,22,23}$ Although three tumors had a THBS1-ALK fusion, only case 13 also showed distinct cytoplasmic membrane staining, albeit focal.
Haimes et al. ${ }^{23}$ also had three tumors with a THBS1$A L K$ fusion, none of which demonstrated this unique staining pattern. Interestingly, smooth cytoplasmic staining was only noted in the two tumors, where no fusion was detected, but specimen quality was optimal for testing (cases 5 and 6). As identification of fusion partners in uterine inflammatory myofibroblastic tumors has not been extensively reported, additional studies are warranted to determine the correlation between ALK staining pattern/localization and specific gene fusions.

Desmin and CD10 were frequently expressed in our series of uterine inflammatory myofibroblastic tumors, which in conjunction with morphology, often poses a diagnostic challenge with smooth muscle and endometrial stromal tumors, respectively. However, the overall morphology of inflammatory myofibroblastic tumors is very different from endometrial stromal tumors. Caldesmon was notably expressed in a small subset of tumors, which further confounds the diagnosis with smooth muscle tumors. The differential diagnosis with regard to both morphology and immunohistochemistry has been thoroughly discussed, ${ }^{18}$ but suffice it to say that spindled, myxoid, or epithelioid tumors associated with a lymphoplasmacytic inflammatory infiltrate should not only be tested for smooth muscle markers, but also for ALK as a part of the initial work-up. Although rare myxoid leiomyosarcomas have shown ALK expression by immunohistochemistry, when fluorescence in situ hybridization was performed, an $A L K$ rearrangement has not been detected. $^{20}$

In prior studies, fluorescence in situ hybridization detected $A L K$ rearrangements in $79 \%$ of inflammatory myofibroblastic tumors, ${ }^{14,17,18,20-23}$ which is nearly identical to the rate detected in this study $(80 \%)$. One tumor (case 8) was negative for $A L K$ rearrangement, but was morphologically diagnostic of an inflammatory myofibroblastic tumor, expressed ALK by immunohistochemistry, and had a SEC31$A L K$ fusion. False negative ALK results by both in situ hybridization and immunohistochemistry have been reported for inflammatory myofibroblastic tumors, ${ }^{28-30}$ emphasizing the importance of using multiple modalities for diagnosis. One tumor (case 6), showed an abnormal fluorescence in situ hybridization pattern characterized by single-isolated green 5' $A L K$ signals (in addition to the normal unsplit red-green pairs). To our knowledge, this has not yet been described in inflammatory myofibroblastic tumors, but has been reported in one series of non-small cell lung carcinomas where the authors considered this pattern negative. ${ }^{31}$ In fact, given that the kinase domain of ALK is encoded by the $3^{\prime}$ part of the gene, cells with an unpaired 5' (green) signals likely represent nonfunctional reciprocal fusion products and are not interpreted as positive for an $A L K$ gene rearrangement. In line with the fluorescence in situ hybridization results, targeted RNA sequencing also showed no evidence of $A L K$ 
rearrangements in this case. However, the lack of an $A L K$ fusion in cases 5 and 6 could potentially be a false negative result if the fusion were to involve exons not covered by the primers used in the targeted RNA sequencing assay. Alternatively, it could also be due to another mechanism of $A L K$ overexpression and activation, such as expression of the novel oncogenic $A L K$ isoform caused by a de novo alternative transcription initiation in $A L K$ intron $19\left(A L K^{\mathrm{ATI}}\right){ }^{32}$

In the current series, six fusion partners including SEC31, TPM3, IGFBP5, TIMP3, THBS1, and DES were identified by targeted RNA sequencing. SEC31 and TPM3 have been previously reported in extrauterine inflammatory myofibroblastic tumors, ${ }^{33-37}$ and IGFPB5, TIMP3, and THBS1 in those of uterine origin, ${ }^{21,23}$ but to our knowledge $D E S$ is a novel fusion. DES encodes the desmin protein, which is a muscle-specific, intermediate filament with an $\alpha$-helical coiled-coil rod central region. ${ }^{38,39}$ Desmin integrates the sarcolemma, $\mathrm{Z}$ disk, and nuclear membrane in sarcomeres and regulates sarcomere architecture. Interestingly, the $D E S$ gene is $\sim 2.8 \mathrm{Mb}$ telomeric to the IGFBP5 gene on chromosome $2 \mathrm{q} 35$. Thus, the DES exon $7-A L K$ exon 19 fusion is presumed to result from an intrachromosomal inversion similar to that described with the IGFPB5 exon 1 -ALK exon 19 fusion. ${ }^{23}$

Although statistical analysis was limited due to the small number of cases, only size was shown to be a significant predictor of behavior in our series. Given that two tumors histologically appeared malignant (cases 4 and 8) while the others were banal, we performed a subanalysis to further identify potential predictors of aggressive behavior. After removing the two tumors that were histologically banal but recurred (cases 2 and 9) from the analysis, severe nuclear atypia, lymphovascular invasion, and increased mitotic activity also became predictive of behavior. With these findings, we propose that if an inflammatory myofibroblastic tumor has all of the above features, it should be classified as malignant. The diagnosis of inflammatory myofibroblastic sarcoma was considered in cases 4 and 8 due to the prominent epithelioid cell morphology, but the tumors lacked most of the other diagnostic features including a minor spindle cell component (one 70\% compact, other $30 \%$ ), myxoid predominance (only seen in one), prominent neutrophils (only rare neutrophils were noted), and neither had a RANBP2 or RRBP1 fusion. ${ }^{40,41}$ Therefore, the diagnosis of malignant inflammatory myofibroblastic tumor was rendered for these two tumors.

We also performed a subanalysis between histologically banal tumors that recurred vs the histologically banal ones that did not recur, but once again, only size was found to be a significant predictor. This finding was not surprising as no morphologic features have consistently shown to be predictive of behavior in extrauterine inflammatory myofibroblastic tumors. Thus, although all patients with uterine inflammatory myofibroblastic tumors should be routinely followed to identify late recurrences, close clinical follow-up is advised on those patients with tumors $\geq 8 \mathrm{~cm}$ due to the high potential for aggressive behavior, as shown in both our study and in that of Parra-Herran et al. ${ }^{18}$

In summary, we have described the broad morphologic spectrum of inflammatory myofibroblastic tumors of the uterus including a subset with aggressive behavior. We confirmed the diagnosis with the use of ancillary testing including immunohistochemistry, fluorescence in situ hybridization, and next-generation sequencing. We also proposed the diagnosis of malignant inflammatory myofibroblastic tumors for lesions $\geq 10.5 \mathrm{~cm}$ with severe nuclear atypia, $\geq 18$ mitoses per 10 high-power fields, and lymphovascular invasion. Correct diagnosis of these rare mesenchymal neoplasms of the uterus is crucial as patients with extrauterine and/or recurrent disease can potentially be treated with tyrosine kinase inhibitors.

\section{Acknowledgments}

We want to thank Dr Kelley Carrick (University of Texas Southwestern Medical Center, Dallas, TX, USA), Dr Franto Francis (University of Texas Southwestern Medical Center, Dallas, TX, USA), Dr Lanu Stoddard (New England Pathology Associates, Southbridge, MA, USA), Dr John Chumas (St Charles Hospital, Port Jefferson, NY, USA), Dr Anjula Thomas (Parkway Health Laboratory, Singapore), Dr Carmen Tornos (Stony Brook University Hospital, Stony Brook, NY, USA), Dr Carole Gengler (Institut Universitaire de Pathologie, Lausanne, Switzerland), and Dr James Mize (Inova Fairfax Hospital, Falls Church, VA) for their contribution of cases.

\section{Disclosure/conflict of interest}

The authors declare no conflict of interest.

\section{References}

1 Gleason BC, Hornick JL. Inflammatory myofibroblastic tumours: where are we now? J Clin Pathol 2008;61: 428-437.

2 Coffin CM, Fletcher JA. Inflammatory myofibroblastic tumor. In: Fletcher CDM, Bridge JA, Hogendoorn PCW (eds). World Health Organization Classification of Tumours of Soft Tissue and Bone. IARC Press: Lyon, 2013, pp 83-84.

3 Lovly CM, Gupta A, Lipson D, et al. Inflammatory myofibroblastic tumors harbor multiple potentially actionable kinase fusions. Cancer Discov 2014;4:889-895.

4 Antonescu CR, Suurmeijer AJ, Zhang L, et al. Molecular characterization of inflammatory myofibroblastic tumors with frequent ALK and ROS1 gene fusions and rare novel RET rearrangement. Am J Surg Pathol 2015;39:957-967. 
5 Gilks CB, Taylor GP, Clement PB. Inflammatory pseudotumor of the uterus. Int J Gynecol Pathol 1987;6: 275-286.

6 Abenoza P, Shek YH, Perrone T. Inflammatory pseudotumor of the cervix. Int J Gynecol Pathol 1994;13: 80-86.

7 Kargi HA, Ozer E, Gokden N. Inflammatory pseudotumor of the uterus: a case report. Tumori 1995;81:454-456.

8 Azuno Y, Yaga K, Suehiro Y, et al. Inflammatory myoblastic tumor of the uterus and interleukin-6. Am J Obstet Gynecol 2003;189:890-891.

9 Gucer F, Altaner S, Mulayim N, et al. Invasive inflammatory pseudotumor of uterine cervix: a case report. Gynecol Oncol 2005;98:325-328.

10 Rabban JT, Zaloudek CJ, Shekitka KM, et al. Inflammatory myofibroblastic tumor of the uterus: a clinicopathologic study of 6 cases emphasizing distinction from aggressive mesenchymal tumors. Am J Surg Pathol 2005;29:1348-1355.

11 Shintaku M, Fukushima A. Inflammatory myofibroblastic tumor of the uterus with prominent myxoid change. Pathol Int 2006;56:625-628.

12 Gupta N, Mittal S, Misra R. Inflammatory pseudotumor of uterus: an unusual pelvic mass. Eur J Obstet Gynecol Reprod Biol 2011;156:118-119.

13 Olgan S, Saatli B, Okyay RE, et al. Hysteroscopic excision of inflammatory myofibroblastic tumor of the uterus: a case report and brief review. Eur J Obstet Gynecol Reprod Biol 2011;157:234-236.

14 Fuehrer NE, Keeney GL, Ketterling RP, et al. ALK-1 protein expression and ALK gene rearrangements aid in the diagnosis of inflammatory myofibroblastic tumors of the female genital tract. Arch Pathol Lab Med 2012;136:623-626.

15 Dong YY, Qu CX, Liu YX, et al. Inflammatory myofibroblastic tumor of uterus: report of a case. Zhonghua Bing Li Xue Za Zhi 2012;41:412-413.

16 Kushnir CL, Gerardi M, Banet N, et al. Extrauterine inflammatory myofibroblastic tumor: A case report. Gynecol Oncol Case Rep 2013;6:39-41.

17 Fraggetta F, Doglioni C, Scollo P, et al. Uterine inflammatory myofibroblastic tumor in a 10-year-old girl presenting as polypoid mass. J Clin Oncol 2015;33: e7-e10.

18 Parra-Herran C, Quick CM, Howitt BE, et al. Inflammatory myofibroblastic tumor of the uterus: clinical and pathologic review of 10 cases including a subset with aggressive clinical course. Am J Surg Pathol 2015;39: 157-168.

19 Subbiah V, McMahon C, Patel S, et al. STUMP un"stumped": anti-tumor response to anaplastic lymphoma kinase (ALK) inhibitor based targeted therapy in uterine inflammatory myofibroblastic tumor with myxoid features harboring DCTN1-ALK fusion. J Hematol Oncol 2015;8:66.

20 Parra-Herran C, Schoolmeester JK, Yuan L, et al. Myxoid leiomyosarcoma of the uterus: a clinicopathologic analysis of 30 cases and review of the literature with reappraisal of its distinction from other uterine myxoid mesenchymal neoplasms. Am J Surg Pathol 2016;40:285-301

21 Sholl LM, Do K, Shivdasani P, et al. Institutional implementation of clinical tumor profiling on an unselected cancer population. JCI Insight 2016;1:e87062.

22 Wojcik J. Inflammatory myofibroblastic tumor with marked pseudo-decidual change. United States and
Canadian Academy of Pathology. Available at http:// materials.uscap.org/2017specsurg_h4_55518.asp.

23 Haimes JD, Stewart C, Kudlow BA, et al. Uterine Inflammatory Myofibroblastic Tumors Frequently Harbor ALK Fusions With IGFBP5 and THBS1. Am J Surg Pathol 2017;41:773-780.

24 Coffin CM, Watterson J, Priest JR, et al. Extrapulmonary inflammatory myofibroblastic tumor (inflammatory pseudotumor). A clinicopathologic and immunohistochemical study of 84 cases. Am J Surg Pathol 1995;19: 859-872.

25 Zheng Z, Liebers M, Zhelyazkova B, et al. Anchored multiplex PCR for targeted next-generation sequencing. Nat Med 2014;20:1479-1484.

26 Banet N, Ning Y, Montgomery EA. Inflammatory myofibroblastic tumor of the placenta: a report of a novel lesion in 2 patients. Int J Gynecol Pathol 2015;34: 419-423.

27 Mino-Kenudson M, Chirieac LR, Law K, et al. A novel, highly sensitive antibody allows for the routine detection of ALK-rearranged lung adenocarcinomas by standard immunohistochemistry. Clin Cancer Res 2010;16:1561-1571.

28 Taheri D, Zahavi DJ, Del Carmen Rodriguez M, et al. For staining of ALK protein, the novel D5F3 antibody demonstrates superior overall performance in terms of intensity and extent of staining in comparison to the currently used ALK1 antibody. Virchows Arch 2016;469:345-350.

29 Coffin CM, Patel A, Perkins S, et al. ALK1 and p80 expression and chromosomal rearrangements involving 2 p23 in inflammatory myofibroblastic tumor. Mod Pathol 2001;14:569-576.

30 Choi E, Williamson SR, Montironi R, et al. Inflammatory myofibroblastic tumour of the urinary bladder: the role of immunoglobulin G4 and the comparison of two immunohistochemical antibodies and fluorescence insitu hybridization for the detection of anaplastic lymphoma kinase alterations. Histopathology 2015;67: 20-38.

31 Dai Z, Kelly JC, Meloni-Ehrig A, et al. Incidence and patterns of ALK FISH abnormalities seen in a large unselected series of lung carcinomas. Mol Cytogenet 2012;5:44.

32 Wiesner $\mathrm{T}$, Lee $\mathrm{W}$, Obenauf $\mathrm{AC}$, et al. Alternative transcription initiation leads to expression of a novel ALK isoform in cancer. Nature 2015;526:453-457.

33 Panagopoulos I, Nilsson T, Domanski HA, et al. Fusion of the SEC31L1 and ALK genes in an inflammatory myofibroblastic tumor. Int J Cancer 2006;118:1181-1186.

34 Kinoshita $\mathrm{Y}$, Tajiri $\mathrm{T}$, Ieiri $\mathrm{S}$, et al. A case of an inflammatory myofibroblastic tumor in the lung which expressed TPM3-ALK gene fusion. Pediatr Surg Int 2007;23:595-599.

35 Lawrence B, Perez-Atayde A, Hibbard MK, et al. TPM3ALK and TPM4-ALK oncogenes in inflammatory myofibroblastic tumors. Am J Pathol 2000;157: 377-384.

36 Yamamoto $\mathrm{H}$, Kohashi K, Oda $\mathrm{Y}$, et al. Absence of human herpesvirus-8 and Epstein-Barr virus in inflammatory myofibroblastic tumor with anaplastic large cell lymphoma kinase fusion gene. Pathol Int 2006;56: 584-590.

37 Cook JR, Dehner LP, Collins MH, et al. Anaplastic lymphoma kinase (ALK) expression in the inflammatory myofibroblastic tumor: a comparative 
immunohistochemical study. Am J Surg Pathol 2001;25:1364-1371.

38 Geisler N, Kaufmann E, Weber K. Proteinchemical characterization of three structurally distinct domains along the protofilament unit of desmin $10 \mathrm{~nm}$ filaments. Cell 1982;30:277-286.

39 Medves S, Demoulin JB. Tyrosine kinase gene fusions in cancer: translating mechanisms into targeted therapies. J Cell Mol Med 2012;16:237-248.
40 Marino-Enriquez A, Wang WL, Roy A, et al. Epithelioid inflammatory myofibroblastic sarcoma: An aggressive intra-abdominal variant of inflammatory myofibroblastic tumor with nuclear membrane or perinuclear ALK. Am J Surg Pathol 2011;35:135-144.

41 Lee JC, Li F, Huang HY, et al. ALK oncoproteins in atypical inflammatory myofibroblastic tumours: novel RRBP1-ALK fusions in epithelioid inflammatory myofibroblastic sarcoma. J Pathol 2017;241:316-323.

Supplementary Information accompanies the paper on Modern Pathology website (http://www.nature.com/ modpathol) 